\title{
Microbiology and Immunology
}

\section{Detection of Norovirus and Sapovirus from diarrheic dogs and cats in Japan}

\author{
Takehisa Soma ${ }^{1}$, Osamu Nakagomi ${ }^{2}$, Toyoko Nakagomi ${ }^{2}$ and Masami Mochizuki ${ }^{3}$ \\ ${ }^{1}$ Veterinary Diagnostic Laboratory, Marupi Lifetech, 103 Fushiocho, Ikeda, Osaka 563-0011, ${ }^{2}$ Department of Hygiene and Molecular \\ Epidemiology, Graduate School of Biomedical Sciences, Nagasaki University, 1-12-4 Sakamoto, Nagasaki 852-8523 and ${ }^{3}$ Laboratory of \\ Infectious Diseases, Joint Faculty of Veterinary Medicine, Kagoshima University, 1-21-24, Korimoto, Kagoshima 890-0065, Japan
}

\begin{abstract}
Norovirus (NoV) and sapovirus ( $\mathrm{SaV}$ ) are important causes of human diarrhea. In this study, between 2007 and 2014 fecal samples were collected from 97 dogs and 83 cats with diarrhea and examined to determine the prevalence of $\mathrm{NoV}$ and $\mathrm{SaV}$ infections in Japan. To detect caliciviruses, approximately 300 bases targeting the polymerase gene were amplified using RT-PCR and subjected to phylogenetic and homology analyses. Specific PCR products were obtained from four canine and nine feline samples: two canine and one feline isolate were classified as NoV, two canine isolates as SaV and the remaining eight feline isolates as vesivirus $(\mathrm{VeV})$. The three $\mathrm{NoV}$ isolates were classified into the same clade as that of known canine and feline NoVs; their homologies (75.9-92.3\%) were higher than those with human genogroup IV (GIV) NoVs (59.1-65.9\%). The homology of the feline NoV isolate with previously reported feline NoV isolates was particularly high (91.7-92.3\%). Regarding SaV, the two canine isolates were classified into the same clade as known canine SaVs and their homologies (72.586.5\%) were higher than those with other mammal SaVs (20.7-58.0\%). The eight feline VeV isolates were assumed to be feline calicivirus. The present study is the first report of the presence of NoV-and $\mathrm{SaV}$-infected dogs and cats in Japan. The findings suggest there are species-specific circulations of NoV and SaV among dogs and cats, in Japan.
\end{abstract}

Key words cat, dog, Norovirus, Sapovirus.

$\mathrm{NoV}, \mathrm{SaV}, \mathrm{VeV}$, lagovirus and nebovirus, belong to the family Caliciviridae, which comprises small non-enveloped viruses containing approximately $7.7 \mathrm{~kb}$ singlestranded RNA; both $\mathrm{NoV}$ and $\mathrm{SaV}$ are clinically important enteric pathogens in humans $(1,2)$. Although canine caliciviruses belonging to $\mathrm{VeV}$ have been detected and are widely prevalent in dogs in Japan and Korea, they have not yet been established as an enteric pathogen for dogs (3-6). On the other hand, FCV, which is a VeV, is a well-known respiratory pathogen in cats (7).

NoV is classified into at least five genogroups (GI, GII, GIII, GIV and GV) based on their VP1 sequences (8).
Additional genogroups (GVI and GVII) have recently been proposed (9-11). GI, GII, GIV and GVI are known to infect humans; of these, GII is the most prevalent $(8,9$, $11,12)$. GIV and GVI NoVs have been detected in dogs, whereas GIV NoV has been detected in a cat and a lion $(13,14)$. The major HuNoV genogroup GII was also detected recently in the feces of three dogs (15) and the roles of canine and feline NoVs in public health and their clinical impact have been inferentially discussed $(16,17)$.

$\mathrm{SaV}$ is classified into 14 genogroups (GI-GV) based on VP1 sequences (18). SaV has not yet been detected in cats. Because $\mathrm{SaV}$ has been detected in only a few dogs,

\section{Correspondence}

Takehisa Soma, Veterinary Diagnostic Laboratory, Marupi Lifetech, 103 Fushiocho, Ikeda, Osaka 563-0011, Japan. Tel: +81-72-753-0335; fax: +81-

72-754-2208; email: takehisa-soma@ah.ds-pharma.co.jp

Received 17 September 2014; revised 15 December 2014; accepted 23 December 2014.

List of Abbreviations: CAdV-1, canine adenovirus type 1; CaNoV, canine NoV; CCOV-I, canine coronavirus type I; CCoV-II, canine coronavirus type II; CDV, canine distemper virus; CPV-2, canine parvovirus type 2; FCoV-I, feline coronavirus type I; FCoV-II, feline coronavirus type II; FCV, feline calicivirus; FeNoV, feline NoV; FPLV, feline panleukopenia virus; HuNoV, human NoV; NoV, norovirus; RV, rotavirus; SaV, sapovirus; VeV, vesivirus. 
the relationship between $\mathrm{SaV}$ of canine and human origin has not yet been elucidated in detail; however, the zoonotic potential of $\mathrm{SaV}$ of $\operatorname{dog}$ origins has been preliminarily discussed (16).

Because of their close relationship with humans as companion animals, dogs and cats have attracted special attention with regard to zoonosis; thus, investigation of their epidemiology is meaningful. In this study, we therefore examined the feces of clinical cases for caliciviruses, compared them with known isolates and discuss their diarrheagenic potential.

\section{MATERIALS AND METHODS}

\section{Specimens}

From September 2007 to March 2014, the feces of 97 household dogs and 83 cats with diarrhea were submitted by veterinary clinics throughout Japan to a commercial veterinary laboratory (Marupi Lifetech, Osaka, Japan) for detection of parvovirus. The fecal samples were suspended in sterile saline, the suspensions centrifuged and nucleic acids isolated from the supernatants using a QIAamp DNA Mini Kit (Qiagen, Germantown, MD, USA) according to the manufacture's instructions, after which they were preserved in a freezer. Although this kit is designed for DNA purification, RNA can also be extracted and purified, according to the attached instruction and a previous study (19).

\section{RT-PCR, sequencing and phylogenetic analyses of caliciviruses}

Using a Qiagen One Step RT-PCR kit, caliciviruses were detected by RT-PCR with the p290d-p289d primer pair that has been reported to bind to the polymerase gene of the calicivirus $(14,20)$. This primer pair is expected to amplify a $331 \mathrm{bp}$ fragment of $\mathrm{SaV}$ and $\mathrm{VeV}$ and a $319 \mathrm{bp}$ fragment of NoV. Two point five $\mu \mathrm{L}$ of the extracted RNA was added to $25 \mu \mathrm{L}$ reaction mixtures containing $5 \mu \mathrm{L}$ of $5 \times$ buffer (finally $1.5 \mathrm{mM} \mathrm{MgCl}$ ), $0.4 \mathrm{mM}$ of deoxynucleoside triphosphate, $10 \mathrm{U}$ RNase inhibitor (Promega, Madison, WI, USA), $0.8 \mu \mathrm{M}$ of the primers and $1.0 \mu \mathrm{L}$ of enzyme mix. The RNA was reverse transcribed at $50^{\circ} \mathrm{C}$ for $30 \mathrm{~min}$, followed by inactivation of reverse transcriptase and denaturation of cDNA template at $95^{\circ} \mathrm{C}$ for $15 \mathrm{~min}$. The cDNA was amplified in 40 sequential cycles of denaturation at $94^{\circ} \mathrm{C}$ for $1 \mathrm{~min}$, annealing at $49^{\circ} \mathrm{C}$ for $1 \mathrm{~min}$ and extension at $72^{\circ} \mathrm{C}$ for $1 \mathrm{~min}$, followed by a final extension at $72^{\circ} \mathrm{C}$ for $7 \mathrm{~min}$. The PCR products were electrophoresed at $100 \mathrm{~V}$ for $35 \mathrm{~min}$ in a $2 \%$ agarose gel, after which they were stained with ethidium bromide and visualized under UV illumination.
The PCR amplicons were purified using ExoSAP-IT, and submitted to direct sequencing of both strands by a dye-terminator cycle sequencing methodology (Bio Matrix Research, Chiba, Japan). The sequences were aligned with reference strains in the GenBank Database using Clustal W and MEGA 6 software (21). Phylogenetic trees were constructed employing the neighborjoining method (22), and their homologies were using BLAST search.

\section{Examination of other diarrheagenic viruses}

To investigate other diarrheagenic viruses in the canine samples, CPV-2 (23), CCoV-I and -II (24), CDV (25), CAdV-1 (26) and RV (27) were examined by PCR. For the feline samples, FPLV (23), CPV-2, FCoV-I and -II (24) and RV were examined.

\section{RESULTS}

\section{Detection of caliciviruses by RT-PCR and their classification into specific genuses}

The PCR products (319 or $331 \mathrm{bp)}$ amplified with the p290d-p289d primer pair were detected in four and nine of 97 canine and 83 feline diarrheal samples, respectively. When the nucleic acid sequences of the PCR products of these 13 samples were subjected to phylogenetic analysis, two canine and one feline sample were classified into the clade of NoV, two canine samples were classified into the clade of $\mathrm{SaV}$, and the other eight feline samples were classified into the clade of $\mathrm{VeV}$, as shown in Figure 1.

\section{Phylogenetic analyses of NoV isolates}

The partial RdRp sequences of each viral genus were compared with the reference strains in the GenBank database. As shown in Figure 1, the dog-derived NoV isolates (Ca/Osaka72/2014/JP and Ca/Aichi595/2013/ JP) and cat-derived NoV isolate (Fe/Saitama382/2013/ JP) were classified into the same clade as known CaNoV and $\mathrm{FeNoV}$, showing 75.9-92.3\% homologies. This homology rate is higher than that (59.1-65.9\%) with GIV HuNoV, which clusters in a different clade on the phylogenetic tree. In addition, the cat-derived isolate (Fe/Saitama382/2013/JP) had a very high homology (91.7-92.3\%) with known FeNoV strains (CU081210E and Pistoia1387). In contrast, the homologies of the canine isolates in the present study with each previous $\mathrm{CaNoV}$ isolate in the same clade were $77.3-91.8 \%$, whereas those with $\mathrm{Ca} / \mathrm{HKU} \_\mathrm{Ca} 035 \mathrm{~F}$ and $\mathrm{Ca} / \mathrm{HKU}_{-}$ Ca026F not classified into the same clade had low homologies (53.6-57.9\%). 


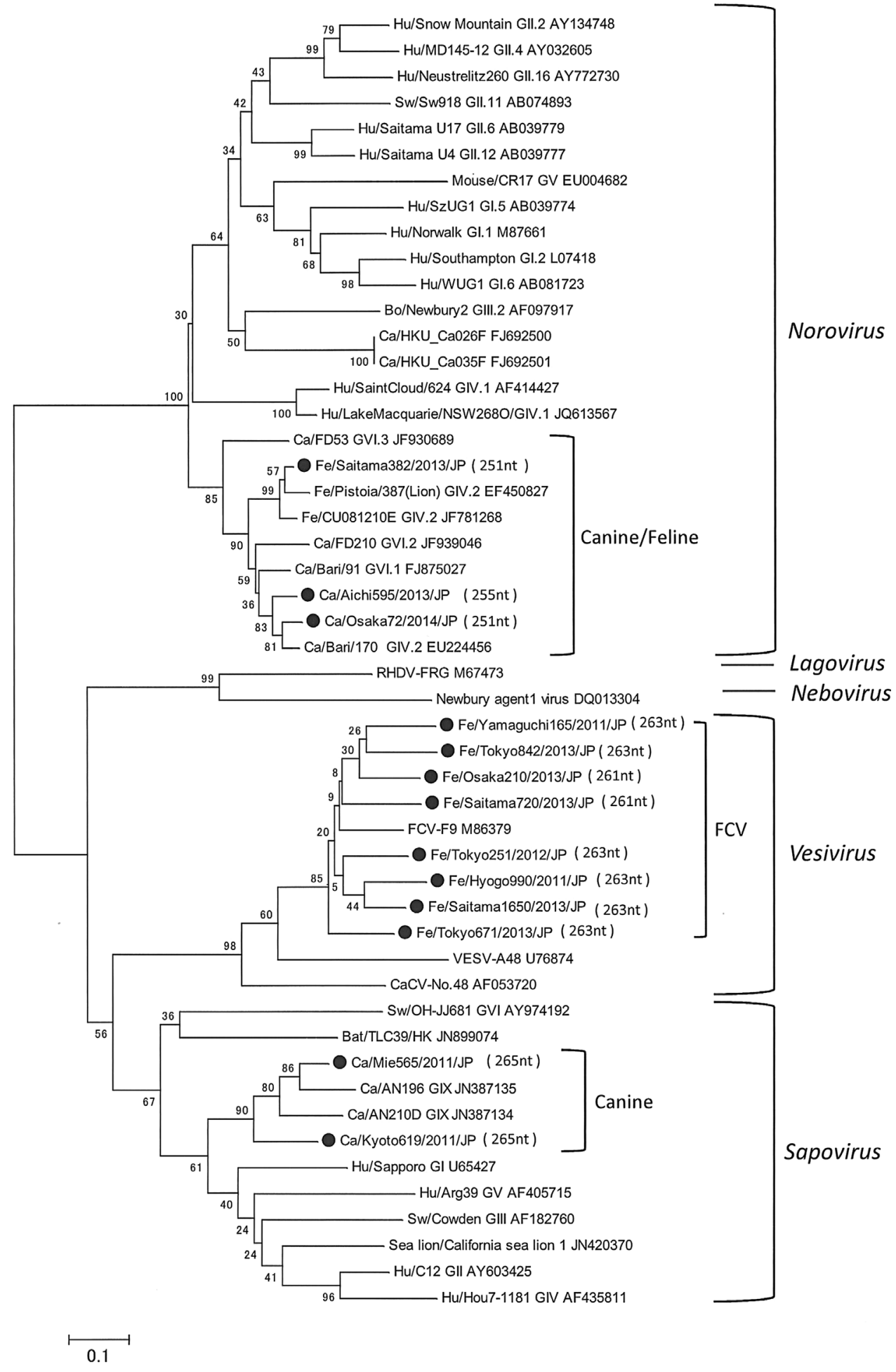

Fig. 1. Phylogenetic tree based on the partial polymerase nucleotide sequences of caliciviruses obtained by the neighbor-joining method with MEGA 6 software. Bootstrap analysis was performed with 1000 replicates. The black dots represent the 13 strains newly detected in this study. The values in parentheses represent nucleotide numbers of actually analyzed sequences. 


\section{Phylogenetic analyses of SaV and VeV isolates}

Two isolates from dogs classified as $\mathrm{SaV}(\mathrm{Ca} / \mathrm{Kyoto619} /$ 2011/JP and $\mathrm{Ca} / \mathrm{Mie} 565 / 2011 / \mathrm{JP}$ ) clustered into the same clade in the phylogenetic tree as previously isolated $\mathrm{CaSaVs}$ in CA, USA (Ca/AN196 and Ca/AN210D) (Fig. 1). The homologies among these four $\mathrm{SaV}$ strains of dog origin were $72.5-86.5 \%$, which are higher than those with $\mathrm{SaV}$ isolates from other mammals including humans (20.7-58.0\%). VeVs from the other eight cats were classified as FCV species on the basis of the clustering pattern in the phylogenetic tree (Fig. 1).

\section{Other viruses detected in the calicivirus- positive samples}

When attempts were made to identify other diarrheagenic viruses in the 13 calicivirus-positive samples, CPV2 was detected in all four canine samples (Ca/Osaka72/ 2014/JP, Ca/Aichi595/2013/JP, Ca/Kyoto619/2011/JP and $\mathrm{Ca} / \mathrm{Mie} 565 / 2011 / \mathrm{JP}), \mathrm{CCoV}-\mathrm{I}$ and -II were detected in two canine samples each, and a mixed infection with both types was noted in one sample (Ca/Osaka72/ 2014/JP) (Table 1). FPLV was detected in one feline sample in which NoV had been detected (Fe/ Saitama382/2013/JP) and in four of the eight feline samples in which VeV had been detected. FCoV-I was detected in six samples (Fe/Yamaguchi165/2011/JP, Fe/Saitama720/2013/JP, Fe/Saitama1650/2013/JP, Fe/ Hyogo990/2011/JP, Fe/Tokyo251/2012/JP and Fe/ Osaka210/2013/JP). None of these five viruses was detected in one sample (Fe/Tokyo671/2013/JP) (Table 2).

\section{DISCUSSION}

Both $\mathrm{NoV}$ and $\mathrm{SaV}$, two isolates of each, were detected in the feces of dogs and one isolate of $\mathrm{NoV}$ was detected in the feces of a cat. To the best of our knowledge, these are the first such findings in Japan and only a few studies have detected $\mathrm{CaSaV}$ and $\mathrm{FeNoV}$ in other parts of the world $(14,28)$.
In the present study, GIV and GVI NoVs derived from canines and felines could not be discriminated by phylogenetic analysis, presumably because only a short part of the polymerase gene was analyzed; however, three NoV isolates detected in dogs and a cat (Ca/Osaka72/ 2014/JP, Ca/Aichi595/2013/JP and Fe/Saitama382/2013/ JP) were classified into the same clade with known CaNoVs and FeNoVs in the phylogenetic tree. In addition, excluding the strains isolated from Chinese dogs (Ca/HKU_Ca035F and Ca/HKU_Ca026F) whose genogroups have not yet been identified (29), the homologies of these three isolates with known $\mathrm{CaNoV}$ and $\mathrm{FeNoV}$ (75.9-92.3\%) were higher than those with GIV HuNoV (59.1-65.9\%), indicating that the present three isolates form a clade with known $\mathrm{CaNoVs}$ and FeNoVs that differs from that of HuNoVs in the phylogenetic tree. It has been suggested that NoV has been circulating among dogs (30); the findings of the present study support this, as described above. However, the homologies of the two NoV isolates with known CaNoVs ranged from 77.3 to $91.8 \%$ even within the same clade, suggesting the presence of several lineages within the $\mathrm{CaNoV}$ genogroups, as described previously $(9,10)$. Although only a small number of FeNoV strains have been characterized, the homologies observed between the present feline NoV isolate (Fe/Saitama382/2013/JP) and previous cat- and lion-derived isolates (CU081210E and Pistoia1387) were extremely high (91.7-92.3\%), suggesting the presence of a unique circulation of $\mathrm{NoV}$ in the Felidae that differs from that in dogs.

On the other hand, the $\mathrm{SaV}$ isolates from dogs $(\mathrm{Ca} /$ Kyoto619/2011/JP and Ca/Mie565/2011/JP) were classified into the same clade as two canine strains previously reported in the USA and the homologies among these (72.5-86.5\%) were markedly higher than those in other mammals (20.7-58.0\%), suggesting the presence of a unique circulation of $\mathrm{SaV}$ in dogs or the Canidae. $\mathrm{CaNoV}, \mathrm{FeNoV}$ and $\mathrm{CaSaV}$ may have an infection cycle in each animal species or family. However, the potential for human infection by some types of $\mathrm{NoV}$ and $\mathrm{SaV}$ in dogs and cats cannot be ruled out, because of the following observations: (i) GII NoV, which is the most

Table 1. Detection of other diarrheagenic viruses in the calicivirus RNA-positive dogs

\begin{tabular}{|c|c|c|c|c|c|c|c|c|c|c|c|}
\hline \multirow[b]{2}{*}{ Isolate } & \multirow[b]{2}{*}{ Collection date } & \multirow[b]{2}{*}{ Breed } & \multirow[b]{2}{*}{ Sex } & \multirow[b]{2}{*}{ Age } & \multirow[b]{2}{*}{ Calicivirus } & \multirow[b]{2}{*}{ CPV-2 } & \multicolumn{2}{|c|}{$\mathrm{CCoV}$} & \multirow[b]{2}{*}{ CDV } & \multirow[b]{2}{*}{ CAdV-1 } & \multirow[b]{2}{*}{ RV } \\
\hline & & & & & & & Type I & Type II & & & \\
\hline Ca/Osaka72 /2014/JP & Feb 2014 & Poodle & Male & 2 months & Norovirus & + & + & + & - & - & - \\
\hline Ca/Aichi595 /2013/JP & Jun 2013 & Borzoi & Female & 2 months & Norovirus & + & - & + & - & - & - \\
\hline Ca/Kyoto619/2011/JP & Jun 2011 & Dachshund & Female & 2 months & Sapovirus & + & - & - & - & - & - \\
\hline Ca/Mie565 /2011/JP & Sep 2011 & Dachshund & Female & 2 months & Sapovirus & + & + & - & - & - & - \\
\hline
\end{tabular}

+ , detected; -, not detected. 
Table 2. Detection of other diarrheagenic viruses in the calicivirus RNA-positive cats

\begin{tabular}{|c|c|c|c|c|c|c|c|c|c|c|}
\hline \multirow[b]{2}{*}{ Isolate } & \multirow[b]{2}{*}{ Collection date } & \multirow[b]{2}{*}{ Breed } & \multirow[b]{2}{*}{ Sex } & \multirow[b]{2}{*}{ Age } & \multirow[b]{2}{*}{ Calicivirus } & \multirow[b]{2}{*}{ FPLV } & \multirow[b]{2}{*}{ CPV-2 } & \multicolumn{2}{|c|}{ FCoV } & \multirow[b]{2}{*}{$\mathrm{RV}$} \\
\hline & & & & & & & & Type I & Type ॥ & \\
\hline Fe/Saitama382 /2013/JP & May 2013 & Crossbreed & Female & 6 months & Norovirus & + & - & - & - & - \\
\hline Fe/Yamaguchi165/2011/JP & Jun 2011 & Crossbreed & Female & 10 years & Vesivirus & - & - & + & - & - \\
\hline Fe/Saitama720 /2013/JP & Jul 2013 & Crossbreed & Male & 1 month & Vesivirus & + & - & + & - & - \\
\hline Fe/Tokyo671 /2013/JP & Mar 2013 & Crossbreed & Male & 16 years & Vesivirus & - & - & - & - & - \\
\hline Fe/Hyogo990 /2011/JP & Dec 2011 & Crossbreed & Male & 1 month & Vesivirus & + & - & + & - & - \\
\hline Fe/Saitama1650/2013/JP & May 2013 & Scottish Fold & Male & 2 months & Vesivirus & - & - & + & - & - \\
\hline Fe/Tokyo251 /2012/JP & Nov 2012 & Somali & Female & 3 years & Vesivirus & + & - & + & - & - \\
\hline Fe/Tokyo842 /2013/JP & Apr 2013 & Crossbreed & Female & 1 year & Vesivirus & + & - & - & - & - \\
\hline Fe/Osaka210 /2013/JP & Jun 2013 & Crossbreed & Male & 1 month & Vesivirus & - & - & + & - & - \\
\hline
\end{tabular}

+ , detected; -, not detected.

prevalent human NoV, has been detected from dogs (15); (ii) GIV and GVI NoVs, which are the main genogroups in dogs and cats, have been detected in humans $(9,11)$; and (iii) antibodies against GVI NoV have been detected in human sera (17).

Although a significantly high detection rate of $\mathrm{CaNoV}$ in dogs with diarrhea has previously been reported (11), epidemiologic data have been insufficient and the pathogenic potential of $\mathrm{NoV}$ and $\mathrm{SaV}$ for dogs and cats remains undetermined. In the present study, we were unable to clarify the pathogenicity of NoVs and $\mathrm{SaVs}$ in dogs and cats, because mixed infections with CPV-2 or FPLV, which are the most virulent causes of diarrhea in dogs and cats, were noted in all five samples in which NoV or $\mathrm{SaV}$ was detected. In previous studies, most dogs infected with $\mathrm{CaNoV}$ were approximately 2-3 months old $(13,30)$. A high CaNoV prevalence has been reported in kennels (31). All four dogs in which NoV and $\mathrm{SaV}$ were detected in the present study were also 2 months old and purebred, suggesting that $\mathrm{CaNoV}$ and $\mathrm{CaSaV}$ infections likely also occur in environments where puppies and kittens are ordinarily kept, such as pet shops and breeding colonies.

FCV was detected in $9.6 \%$ of fecal specimens from cats in the present study. Although FCV is a representative pathogen for upper respiratory tract, it has been demonstrated not only in laryngopharyngeal secretions but also in feces (32). Although FCV, which replicates in the upper respiratory epithelial cells, potentially passes through the alimentary tract and is excreted in the feces, a difference in resistance to bile salts has been observed between FCV isolates from respiratory secretions and feces, suggesting the presence of enterocyte-infecting FCV (32). No other diarrheagenic viruses were detected in one FCV-positive sample (Fe/Tokyo671/2013/JP), and only FCoV-I, which generally causes no or mild symptoms of intestinal infections when it infects alone, was detected in three samples (Fe/Yamaguchi165/2011/ JP, Fe/Saitama1650/2013/JP and Fe/Osaka210/2013/JP). These findings suggest that some type(s) of FCV may be enteric pathogens in cats, but this remains to be elucidated in future studies, including experimental infections.

\section{DISCLOSURE}

No authors have conflicts of interest to declare regarding this study.

\section{REFERENCES}

1. Glass R.I., Parashar U.D., Estes M.K. (2009) Norovirus gastroenteritis. N Engl J Med 361: 1776-85.

2. Bank-Wolf B.R., König M., Thiel H.J. (2010) Zoonotic aspects of infections with noroviruses and sapoviruses. Vet Microbiol 140: 204-12.

3. Mochizuki M., Kawanishi A., Sakamoto H., Tashiro S., Fujimoto R., Ohwaki M. (1993) A calicivirus isolated from a dog with fatal diarrhoea. Vet Rec 132: 221-22.

4. Matsuura Y., Tohya Y., Nakamura K., Shimojima M., Roerink F., Mochizuki M., Takase K., Akashi H., Sugimura T. (2002) Complete nucleotide sequence, genome organization and phylogenetic analysis of the canine calicivirus. Virus Genes 25: 67-73.

5. Mochizuki M., Hashimoto M., Roerink F., Tohya T., Matsuura Y., Sasaki N. (2002) Molecular and seroepidemiological evidence of canine calicivirus infections in Japan. J Clin Microbiol 40: $2629-31$.

6. Jang H.K., Tohya Y., Han K.Y., Kim T.J., Son C.S., Mochizuki M. (2003) Seroprevalence of canines calicivirus and canine minute virus in the Republic of Korea. Vet Rec 153: 150-52.

7. Radford A.D., Coyne K.P., Dawson S., Porter C.J., Gaskell R.M. (2007) Feline calicivirus. Vet Res 38: 319-35.

8. Zheng D.P., Ando T., Fankhauser R.L., Beard R.S., Glass R.I., Monroe S.S. (2005) Norovirus classification and proposed strain nomenclature. Virol 15: 312-23.

9. Martella V., Pinto P., Buonavoglia C. (2011) Canine noroviruses. Vet Clin North Am Small Anim Pract 41: 1171-81. 


\section{T. Soma et al.}

10. Vinjé J. (2014) Advances in Laboratory Methods for Detection and Typing of Norovirus. J Clin Microbiol pii: JCM. 01535-14.

11. Mesquita J.R., Barclay L., Nascimento M.S., Vinjé J. (2010) Novel norovirus in dogs with diarrhea. Emerg Infect Dis 16: 980-2.

12. Hoa Tran, Trainor T.N., Nakagomi E., Cunliffe T., Nakagomi N.A. (2013) Molecular epidemiology of noroviruses associated with acute sporadic gastroenteritis in children: Global distribution of genogroups, genotypes and GII.4 variants. J Clin Virol 56: 185-93.

13. Martella V., Lorusso E., Decaro N., Elia G., Radogna A., D'Abramo M., Desario C., Cavalli A., Corrente M., Camero M., Germinario C.A., Bányai K., Di Martino B., Marsilio F., Carmichael L.E., Buonavoglia C. (2008) Detection and molecular characterization of a canine norovirus. Emerg Infect Dis 14: 1306-8.

14. Pinto P., Wang Q., Chen N., Dubovi E.J., Daniels J.B., Millward L.M., Buonavoglia C., Martella V., Saif L.J. (2012) Discovery and genomic characterization of noroviruses from a gastroenteritis outbreak in domestic cats in the US. PLoS One 32739.

15. Summa M., von Bonsdorff C.H., Maunula L. (2012) Pet dogs-a transmission route for human noroviruses? J Clin Virol 53: 2447 .

16. Humphrey T.J., Cruickshank J.G., Cubitt W.D. (1984) An outbreak of calicivirus associated gastroenteritis in an elderly persons home. A possible zoonosis? J Hyg Lond 93: 293-9.

17. Mesquita J.R., Costantini V.P., Cannon J.L., Lin S.C., Nascimento M.S., Vinjé J. (2013) Presence of antibodies against genogroup VI norovirus in humans. Virol J 10: 176.

18. Scheuer K.A., Oka T., Hoet A.E., Gebreyes W.A., Molla B.Z., Saif L.J., Wang Q. (2013) Prevalence of porcine noroviruses, molecular characterization of emerging porcine sapoviruses from finisher swine in the United States, and unified classification scheme for sapoviruses. J Clin Microbiol 51: 2344-53.

19. Dye C., Helps C.R., Siddell S.G. (2008) Evaluation of real-time RT-PCR for the quantification of FCoV shedding in the faeces of domestic cats. J Feline Med Surg 10: 167-74.

20. Jiang X., Huang P.W., Zhong W.M., Farkas T., Cubitt D.W., Matson D.O. (1999) Design and evaluation of a primer pair that detects both Norwalk- and Sapporo-like caliciviruses by RTPCR. J Virol Methods 83: 145-54.
21. Tamura K., Stecher G., Peterson D., Filipski A., Kumar S. (2013) MEGA6: Molecular Evolutionary Genetics Analysis version 6.0. Mol Biol Evol 30: 2725-9.

22. Saitou N., Nei M. (1987) The neighbor-joining method: A new method for reconstructing phylogenetic trees. Mol Biol Evol 4: $406-25$.

23. Pereira C.A., Monezi T.A., Mehnert D.U., D'Angelo M., Durigon E.L. (2000) Molecular characterization of canine parvovirus in Brazil by polymerase chain reaction assay. Vet Microbiol 75: 127-33.

24. Addie D.D., Schaap I.A., Nicolson L., Jarrett O. (2003) Persistence and transmission of natural type I feline coronavirus infection. J Gen Virol 84: 2735-44.

25. Frisk A.L., König M., Moritz A., Baumgärtner W. (1999) Detection of canine distemper virus nucleoprotein RNA by reverse transcription-PCR using serum, whole blood, and cerebrospinal fluid from dogs with distemper. J Clin Microbiol 37: $3634-43$.

26. Hu R.L., Huang G., Qiu W., Zhong Z.H., Xia X.Z., Yin Z. (2001) Detection and differentiation of CAV-1 and CAV-2 by polymerase chain reaction. Vet Res Commun 25: 77-84.

27. Gouvea V., Glass R.I., Woods P., Taniguchi K., Clark H.F., Forrester B., Fang Z.Y. (1990) Polymerase chain reaction amplification and typing of rotavirus nucleic acid from stool specimens. J Clin Microbiol 28: 276-82.

28. Li L., Pesavento P.A., Shan T., Leutenegger C.M., Wang C., Delwart E. (2011) Viruses in diarrhoeic dogs include novel kobuviruses and sapoviruses. J Gen Virol 92: 2534-41.

29. Tse H., Lau S.K.P., Chan W.M., Choi G.K.Y., Woo P.C.Y., Yuen K.Y. (2012) Complete genome sequences of novel canine noroviruses in Hong Kong. J Virol 86: 9531-2.

30. Ntafis V., Xylouri E., Radogna A., Buonavoglia C., Martella V. (2010) Outbreak of canine norovirus infection in young dogs. J Clin Microbiol 48: 2605-8.

31. Mesquita J.R., Nascimento M.S. (2012) Molecular epidemiology of canine norovirus in dogs from Portugal, 2007-2011. BMC Vet Res 8: 107.

32. Mochizuki M. (1992) Different stabilities to bile among feline calicivirus strains of respiratory and enteric origin. Vet Microbiol 31: 297-302. 